Factors Influencing the Cost-Effectiveness Outcomes of HPV Vaccination and Screening Interventions in Low-to-Middle-Income Countries (LMICs)

Okeah, Bernard O; Ridyard, Colin $\mathrm{H}$
\end{abstract}

\title{
Applied Health Economics and Health Policy
}

DOI:

$10.1007 / s 40258-020-00576-7$

Published: 01/10/2020

Peer reviewed version

Cyswllt i'r cyhoeddiad / Link to publication

Dyfyniad o'r fersiwn a gyhoeddwyd / Citation for published version (APA):

Okeah, B. O., \& Ridyard, C. H. (2020). Factors Influencing the Cost-Effectiveness Outcomes of HPV Vaccination and Screening Interventions in Low-to-Middle-Income Countries (LMICs): A Systematic Review. Applied Health Economics and Health Policy, 18(5), 641-654. https://doi.org/10.1007/s40258-020-00576-7

Hawliau Cyffredinol / General rights

Copyright and moral rights for the publications made accessible in the public portal are retained by the authors and/or other copyright owners and it is a condition of accessing publications that users recognise and abide by the legal requirements associated with these rights.

- Users may download and print one copy of any publication from the public portal for the purpose of private study or research.

- You may not further distribute the material or use it for any profit-making activity or commercial gain

- You may freely distribute the URL identifying the publication in the public portal ?

Take down policy

If you believe that this document breaches copyright please contact us providing details, and we will remove access to the work immediately and investigate your claim. 


\section{Factors influencing the cost-effectiveness outcomes of HPV vaccination and screening interventions in Low-to Middle-Income Countries (LMICs): A systematic review}

\section{Author Information}

\section{$\underline{\text { Affiliations }}$}

School of Healthcare Sciences, Bangor University, Bangor, Gwynedd, LL57 2EF. UK

Bernard O Okeah

Centre for Health Economics and Medicines Evaluation, Bangor University, Normal Site, Bangor, Gwynedd, LL57 2PZ. UK

Colin H Ridyard PhD

$\underline{\text { Contributions }}$

B.O. conceived the idea, designed the study protocol and was the first reviewer. C.R. verified the study protocol, was the second reviewer and supervised the study. The authors discussed the findings of this study and contributed to the final report.

Corresponding author

Correspondence to Colin H Ridyard

E-mail: c.h.ridyard@bangor.ac.uk

Phone: 01248388861 


\title{
Factors influencing the cost-effectiveness outcomes of HPV vaccination and screening interventions in Low-to Middle-Income Countries (LMICs): A systematic review
}

\begin{abstract}
Background: Cervical cancer ranks fourth amongst the commonest malignancies worldwide and the second most prevalent cancer afflicting women in low-to middle-income countries (LMICs), hence, of great public health importance. LMICs are the most affected regions as evidenced by their high prevalence of the disease. Mortality associated with cervical neoplasms is preventable through the implementation of recommended preventive approaches.
\end{abstract}

Aims: This review aimed at appraising evidence on the cost-effectiveness of cervical cancer prevention interventions in LMICs involving cervical screening and HPV vaccination programmes.

Methods: A search of CINAHL, MEDLINE, PubMed, and Web of Science was elicited and studies published between $1^{\text {st }}$ January 2008 and $31^{\text {st }}$ December 2018 were retrieved. Two authors independently undertook the screening, review, selection of studies, and data extraction with disagreements being resolved through discussion and consensus.

Results: Twelve studies were selected. The cost-effectiveness outcomes of HPV vaccination and screening interventions are dependent on age, screening method used, intervention coverage, and the number of doses or visits required for vaccination and screening respectively. A combination of visual inspection with acetic acid (VIA) screening and HPV vaccination appears to be the most cost-effective approach in reducing the lifetime risk for HPV-linked cervical neoplasms. Similarly, vaccination as a stand-alone intervention is potentially cost effective provided the coverage is maintained between $70-100 \%$.

Conclusions: HPV vaccination and screening interventions may be cost-effective in LMICs and potentially reduce the lifetime risk, economic burden, and associated mortality. However, it is important to consider the factors that influence the cost-effectiveness of cervical cancer prevention interventions for better outcomes to be realised.

Key points for decision makers

- HPV vaccination and VIA screening appear to be cost-effective approaches for reducing the lifetime risks for cervical cancer in LMICs

- A high loss-to-follow-up for vaccination and screening is associated with increased mortality

- Early screening for HPV below the age of 30 years could potentially reduce cervical cancer associated morbidity and mortality

- Implementation of organised programmes improves coverage and has better outcomes compared to opportunistic programmes 


\section{Introduction}

Cervical cancer ranks fourth among the more ubiquitous global malignancies and is the second most prevalent cancer afflicting women in low-to middle-income countries (LMICs), thus, representing a significant public health challenge (1). It represents an estimated $6.6 \%$ of all neoplasms in women (2) with over 550,000 new cases and 311,365 fatalities reported in $2018(2,3)$. This figure is higher than the cervical cancer-linked mortality of approximately 266,000 deaths reported in 2012 (4) and 270,000 deaths recorded in 2015 (5).

LMICs have a higher burden of cervical carcinoma recording more than two thirds of global cases and $90 \%$ of all associated deaths (6). Future projections suggest a potential rise in cancer cases amongst women by $0.11 \%$ in 2035 while that of males will drop by $0.03 \%$ (7). Despite these facts, countries bearing the highest burden of cervical carcinomas utilize only $5 \%$ of all resources spent on the disease (5).

Healthcare systems in most LMICs are fragmented and present many challenges which limit women's access to treatment interventions (5). Many countries lack well organized programmes for cervical cancer mitigation with financial constraints further limiting access to relevant interventions; thus, , causing up to an 18 times greater mortality rate in comparison to developed countries (3). Cervical cancer propagates the cycle of poverty and undermines economic development in affected communities.

An association exists between human papilloma virus (HPV) infections and cervical cancer (8), which account for $15.4 \%$ of all carcinomas linked to infections within subSaharan Africa (9). There exists clinically effective vaccines against the oncogenic strains of HPV (10) but these are not freely available in most LMICs (6). Lack of government prioritization in LMICs limits access to life saving preventive interventions for women. 
Early detection of cervical neoplasms is a major prognostic factor and improves women's survival rate (11). Deaths and disability occasioned by cervical cancer are preventable through multidisciplinary and comprehensive interventions delivered across the lifespan to ensure their cost-effectiveness $(5,12,13)$. The WHO's comprehensive approach to controlling cervical carcinoma provides for primary, secondary, and tertiary prevention measures such as vaccination, screening, and post-treatment follow-up services respectively $(12,14)$.

A quick scoping of existing literature on the current research question retrieved two systematic reviews undertaken in LMICs. The first review (15) mainly focussed on HPV vaccination with minimal attention paid to screening interventions. Notably, the conclusions made by Fesenfeld, Hutubessy, and Jit, (2013) only centred around the implications of model types, vaccine prices, and vaccinating boys on cost-effectiveness outcomes. A second systematic review (16) focussed on how decision models utilized in HPV vaccination programmes account for the apparent challenges in LMICs.

The evidence regarding the most suitable strategies for preventing cervical neoplasms and the specific factors influencing the cost-effectiveness of such interventions is not comprehensive. Notably, the preferences for screening or vaccination approaches varies across LMICs and consistency in practice appears to be lacking. There is therefore a need to consolidate evidence on the interventions and optimize resource allocation for preventing cervical neoplasms in LMICs.

This systematic review (SR) comparatively investigates the factors influencing the costeffectiveness outcomes of both screening and vaccination interventions. Furthermore, the review appraises the available economic evidence on the cost-effectiveness of HPV screening programmes in LMICs not addressed by previously undertaken studies. Lastly, the 
researchers explore the cost-effectiveness for combining various HPV screening approaches with HPV vaccination in LMICs. The findings are of significance to health policy formulators as well as health practitioners involved in mitigation of cervical carcinomas.

\section{Research questions}

1. What factors influence the cost-effectiveness outcomes of HPV vaccination and screening in LMICs?

2. Are HPV screening interventions cost-effective in LMICs?

3. Which screening approach is the most cost-effective when combined with HPV vaccination in LMICs? 


\section{Methods}

The study adopted a systematic review design in answering the research questions. The reporting was undertaken in compliance with the PRISMA (Preferred Reporting Items for Systematic Reviews and Meta-Analyses) statement (17). A protocol for this systematic review is available on request through the authors' email addresses (bernardokeah@gmail.com or c.h.ridyard@bangor.ac.uk).

Table 1 below outlines the PICOs (Population, Intervention, Control, Outcomes) framework that was adopted to facilitate a literature search across databases using appropriate medical subject headings (MeSH) (18). The review targeted cost-effectiveness studies assessing HPV vaccination and screening in LMICs. Grey literature and unpublished studies were excluded from this review to avoid their potential effect on results synthesis (19).

Table 1: PICOS framework

\begin{tabular}{|l|l|}
\hline Population & $\begin{array}{l}\text { The population for the HPV vaccination comprised of Girls and boys from } \\
\text { (low-to-middle income countries (LMICs) aged between 9-15 years. The } \\
\text { population for cervical cancer screening comprised of women from LMICs } \\
\text { aged between 20-69 years }\end{array}$ \\
\hline Intervention & $\begin{array}{l}\text { HPV vaccination and/or routine screening through visual inspection with } \\
\text { acetic acid (VIA), cytology, or Pap smear }\end{array}$ \\
\hline Control & No intervention or vaccination only, or screening only. \\
\hline Outcomes & $\begin{array}{l}\text { i. } \quad \begin{array}{l}\text { Reduction in the incidence and mortality associated with cervical } \\
\text { neoplasms }\end{array} \\
\text { ii. } \quad \begin{array}{l}\text { Incremental Cost-Effectiveness Ratio (ICER) } \\
\text { iii. Incremental costs. }\end{array}\end{array}$ \\
\hline $\begin{array}{l}\text { Study } \\
\text { Design }\end{array}$ & \begin{tabular}{l} 
Cost-effectiveness analysis studies \\
\hline
\end{tabular}
\end{tabular}

CINAHL, MEDLINE, PubMed, and Web of Science were searched between $15^{\text {th }}$ July 2019 and $25^{\text {th }}$ July 2019. Only studies published in English, between $1^{\text {st }}$ January 2008 and $31^{\text {st }}$ December 2018 were included. Search terms applied included developing countr*, lowincome countr*, screen*, monitor*, check*, VIA, cervical cancer, cervical neoplasm*, HPV, 
vaccine*, cost-effective* and cost-utility. Boolean operators ' $O \mathrm{R}^{*}$ ' and truncation $\left.{ }^{*}\right)$ to were useful in expanding the search whereas "AND" was utilized in limiting the searches. Appendix 1 below provides the full search strategy and the limiters as applied in the MEDLINE database.

Eligibility assessment commenced with the screening of the article titles after which the abstract and the entire article were read to ascertain consistency with the inclusion criteria outlined in the PICOS framework. The selection process also entailed an assessment of the methodological rigour of the studies.

A Microsoft excel data extraction sheet was piloted using two randomly selected articles and refined based on the pilot outcome $(20,21)$. The extraction process followed a systematic process to minimize errors and enhance the quality of the findings. The extracted data was checked against the checklist and inclusion criteria to ensure its completeness and accuracy.

Information extracted included the type of economic evaluation undertaken, the study perspective as well as the key assumptions. Additional data included the study population, the interventions, and the nature of the study whether model-based or empirical studies. The extraction also included the source of the cost measures, costing approach, the reference year, type of costs included, benefits accrued, sensitivity analysis undertaken, the costeffectiveness threshold, the ICERs, the study conclusions, and the declaration of funding as well as any conflicts of interest.

The Drummond and the CHEC (consensus on health economics criteria) checklists were employed in assessing the risk of bias $(\mathrm{RoB})(20,22)$. The RoB was useful in ascertaining any significant variations with respect to the cost-effectiveness of the interventions. The assessment also focussed on the transparency and clarity in reporting cost components (23). Following the RoB assessment, studies were excluded based on their 
transparency. Studies that did not report on the relevant parameters as outlined in the CHEC checklist were deemed as lacking transparency and excluded from the review.

Summary measures included the incremental costs for HPV vaccination and cervical cancer screening as well as the ICER (24). Additional measures included the benefits reported across studies such as the effect of the interventions on the lifetime risk as well as the mortality linked with cervical carcinomas.

Two authors independently undertook the screening, review, selection of studies, and data extraction with disagreements resolved through discussion and consensus. This review provides a narrative synthesis of the results and does not constitute a meta-analysis of the study findings. The costs and benefits are reported in a uniform currency of international dollars (I\$) by applying the purchasing power parity exchange rates (25) and discounted at $3 \%$ to 2017 as the base year. 


\section{Results}

\section{Study selection}

The figure 1 below summarizes the process for identifying, screening, and selecting of studies for the present systematic review

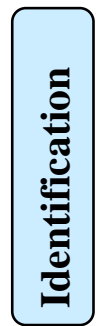

Records retrieved from CINAHL, MEDLINE, PubMed, and Web of Science $(\mathrm{n}=182)$

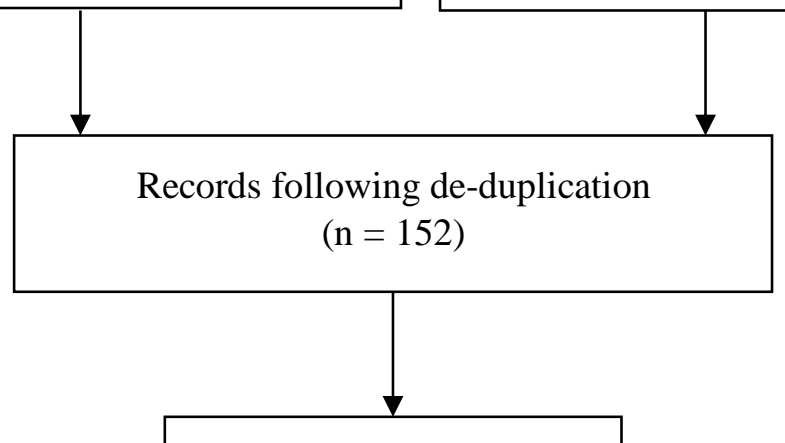

Additional articles identified from searching bibliographies $(n=6)$

\begin{tabular}{|c|c|}
\hline $\begin{array}{l}\text { Record titles screened } \\
\qquad(\mathrm{n}=152)\end{array}$ & $\begin{array}{l}\text { Excluded irrelevant titles } \\
\qquad(\mathrm{n}=114)\end{array}$ \\
\hline$\downarrow$ & Excluded records due to \\
\hline $\begin{array}{l}\text { Abstracts assessed using } \\
\text { eligibility criteria } \\
(\mathrm{n}=38)\end{array}$ & $\begin{array}{l}\text { irrelevant design }(\mathrm{n}=10), \\
\text { or inappropriate study } \\
\text { population }\end{array}$ \\
\hline $\begin{array}{l}\text { Reading of full-text } \\
\text { articles } \\
(\mathrm{n}=27)\end{array}$ & $\begin{array}{l}\text { Excluded articles, due to } \\
\text { risk of bias }(n=3) \text {, high- } \\
\text { income country/ multi- }\end{array}$ \\
\hline $\begin{array}{c}\text { Studies included for final } \\
\text { synthesis } \\
(\mathrm{n}=12)\end{array}$ & $\begin{array}{l}\text { country results }(n=5) \text {, or } \\
\text { incomplete outcome } \\
\text { measures }(n=7)\end{array}$ \\
\hline
\end{tabular}

Figure 1: PRISMA flow chart summarizing the process for selecting studies 
The literature search yielded 188 studies and 152 studies after deduplication. Following title review, 114 studies were excluded whereas a further 11 articles were discarded following abstract review. Twelve articles were excluded after full text reading and a further three articles excluded after assessing the RoB, reporting transparency, as well as their transferability. Finally, this review included twelve (12) studies that met the stated criteria.

\section{Study characteristics}

The review provided eleven cost-effectiveness analysis (CEA) studies and one cost-utility analysis (CUA) study published. All the retrieved studies originated from LMICs namely Thailand, Colombia, Uganda, Laos, Malaysia, Iran, China, Honduras, India, South Africa, and Brazil as shown in Figure 2. All the retrieved studies were model-based and relied on published data for model calibration.

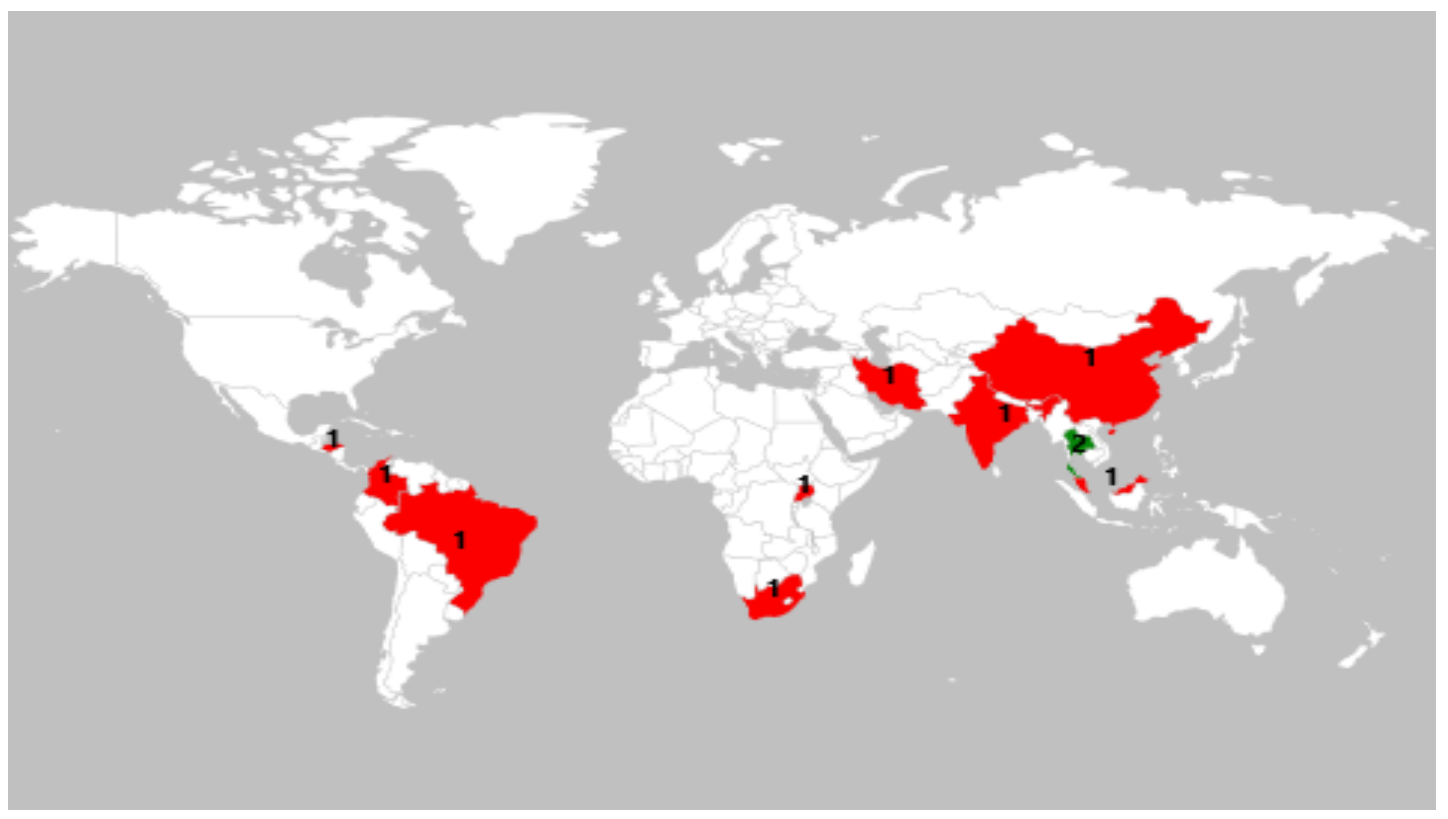

Figure 2: Distribution of selected studies per country

The studies were modelled on hypothetical participants and their age ranges depended on the intervention type. The participants included females aged 9-15 years for the HPV vaccination interventions and 21-69 years for screening interventions while one study 
explored the potential benefit of vaccinating boys. The interventions reported in the included studies were HPV vaccination in combination with screening $(8,26-29)$, HPV vaccination only (30-33), and screening only (34-36).

The outcomes of the interventions reported include a reduction in the cervical carcinoma incidence, improvement in the quality of life and mortality reduction. The authors reported the cost-effectiveness of the interventions by comparing the ICERs with the per capita GDP per capita as proposed by the WHO's Commission for Macroeconomics and Health (37-39). Interventions whose ICERs fall below the per capita GDP (1XGDP per capita) are highly cost-effective while those that do not surpass two times the GDP (2XGDP) are very cost-effective. Lastly, interventions whose ICERs fall below three times the per capita GDP (3XGDP per capita) are considered cost-effective. Based on these measures, four of the interventions were highly cost-effective whereas half of the interventions surpassed the cost-effectiveness threshold, while two did not. 
Table 2: Summary of study characteristics

\begin{tabular}{|c|c|c|c|c|c|c|c|c|c|c|c|}
\hline Author & Country & Population & Age & Intervention & $\begin{array}{l}\text { Empirical- } \\
\text { or model- } \\
\text { based EE } \\
\end{array}$ & Comparator(s) & $\begin{array}{c}\text { Outcome } \\
\text { measure(s) }\end{array}$ & Perspective & $\begin{array}{c}\text { Intervention } \\
\text { cost- } \\
\text { effectiveness }\end{array}$ & $\begin{array}{c}\text { Competi } \\
\text { ng } \\
\text { interests } \\
\end{array}$ & Funding \\
\hline $\begin{array}{l}\text { Andres-Gamboa et } \\
\text { al. (34) }\end{array}$ & Colombia & Girls & $\begin{array}{l}21- \\
69\end{array}$ & $\begin{array}{l}\text { Screening: } \\
\text { Cytology or } \\
\text { HPV DNA }\end{array}$ & Model & No screening & YLS & $\begin{array}{l}\text { Payers } \\
\text { perspective }\end{array}$ & $\begin{array}{l}\text { Cost- } \\
\text { effective }\end{array}$ & $\begin{array}{l}\text { None } \\
\text { declared }\end{array}$ & $\begin{array}{l}\text { Not } \\
\text { stated }\end{array}$ \\
\hline Campos et al., (35) & Uganda & Women & $>35$ & $\begin{array}{l}\text { Screening: } \\
\text { VIA or HPV } \\
\text { DNA }\end{array}$ & Model & No screening & YLS & Societal & $\begin{array}{l}\text { Cost- } \\
\text { effective }\end{array}$ & Declared & $\begin{array}{l}\text { NCI and } \\
\text { B\&MGF }\end{array}$ \\
\hline $\begin{array}{l}\text { Chanthavilay et al. } \\
\text { (28) }\end{array}$ & Laos & $\begin{array}{l}\text { Women } \\
\text { Girls }\end{array}$ & 10 & $\begin{array}{l}\text { Vaccination } \\
\text { plus } \\
\text { screening }\end{array}$ & Model & $\begin{array}{l}\text { Screening only } \\
\text { or vaccination } \\
\text { only }\end{array}$ & DALYs & $\begin{array}{l}\text { Public } \\
\text { healthcare }\end{array}$ & $\begin{array}{l}\text { Cost- } \\
\text { effective }\end{array}$ & Declared & AUF \\
\hline Ezat and Aljunid (8) & Malaysia & Women & $>18$ & $\begin{array}{l}\text { Vaccination } \\
\text { plus } \\
\text { screening }\end{array}$ & Model & Screening only & QALYs & $\begin{array}{l}\text { Public } \\
\text { healthcare }\end{array}$ & $\begin{array}{l}\text { Cost- } \\
\text { effective }\end{array}$ & $\begin{array}{l}\text { None } \\
\text { declared }\end{array}$ & $\begin{array}{l}\text { Not } \\
\text { stated }\end{array}$ \\
\hline Khatibi et al. (30) & Iran & Girls & 15 & Vaccination & Model & $\begin{array}{l}\text { No vaccination } \\
\text { no screening }\end{array}$ & QALYs & Government & $\begin{array}{l}\text { Not cost- } \\
\text { effective }\end{array}$ & Declared & $\begin{array}{l}\text { Not } \\
\text { stated }\end{array}$ \\
\hline Levin et al. (36) & China & Women & $\begin{array}{l}35- \\
45\end{array}$ & $\begin{array}{l}\text { Screening: } \\
\text { HPV DNA }\end{array}$ & Model & $\begin{array}{l}\text { Screening: } \\
\text { Cytology }\end{array}$ & YLS & Societal & $\begin{array}{l}\text { Cost- } \\
\text { effective }\end{array}$ & Declared & B\&MGF \\
\hline Molina et al. (33) & Honduras & Girls & 11 & Vaccination & Model & $\begin{array}{l}\text { No vaccination, } \\
\text { no screening }\end{array}$ & DALYs & $\begin{array}{l}\text { Societal and } \\
\text { government }\end{array}$ & $\begin{array}{l}\text { Highly cost- } \\
\text { effective }\end{array}$ & Declared & B\&MGF \\
\hline Prinja et al. (32) & India & Girls & 11 & Vaccination & Model & $\begin{array}{l}\text { Treatment of } \\
\text { unvaccinated }\end{array}$ & QALYs & $\begin{array}{l}\text { Societal and } \\
\text { government }\end{array}$ & $\begin{array}{l}\text { Highly cost- } \\
\text { effective }\end{array}$ & $\begin{array}{l}\text { None } \\
\text { declared }\end{array}$ & $\begin{array}{l}\text { Not } \\
\text { stated }\end{array}$ \\
\hline Sharma et al. (26) & Thailand & Girls & $\begin{array}{l}>9 \\
\text { years }\end{array}$ & $\begin{array}{l}\text { Vaccination } \\
\text { plus } \\
\text { screening }\end{array}$ & Model & Screening & YLS & Societal & $\begin{array}{l}\text { Cost- } \\
\text { effective }\end{array}$ & $\begin{array}{l}\text { None } \\
\text { declared }\end{array}$ & B\&MGF \\
\hline Sinanovic et al. (27) & $\begin{array}{l}\text { South } \\
\text { Africa }\end{array}$ & $\begin{array}{c}\text { Girls } \\
\text { Women }\end{array}$ & $\begin{array}{l}>12 \\
>30\end{array}$ & $\begin{array}{l}\text { Vaccination } \\
\& \text { screening }\end{array}$ & Model & $\begin{array}{l}\text { Screening: } \\
\text { Cytology }\end{array}$ & QALYs & Societal & $\begin{array}{l}\text { Highly cost- } \\
\text { effective }\end{array}$ & Declared & B\&MGF \\
\hline $\begin{array}{l}\text { Termrungruanglert et } \\
\text { al. (31) }\end{array}$ & Thailand & Girls & $>12$ & Vaccination & Model & No vaccination & QALYs & $\begin{array}{l}\text { Healthcare } \\
\text { provider }\end{array}$ & $\begin{array}{l}\text { Cost- } \\
\text { effective }\end{array}$ & Declared & $\begin{array}{l}\text { Not } \\
\text { stated }\end{array}$ \\
\hline Vanni et al. (29) & Brazil & $\begin{array}{c}\text { Girls } \\
\text { Women } \\
\text { Boys } \\
\end{array}$ & $\begin{array}{l}10, \\
25- \\
60 \\
\end{array}$ & $\begin{array}{l}\text { Vaccination } \\
\text { \& screening }\end{array}$ & Model & $\begin{array}{l}\text { Screening: } \\
\text { Cytology }\end{array}$ & QALYs & $\begin{array}{l}\text { Health } \\
\text { system }\end{array}$ & $\begin{array}{l}\text { Cost- } \\
\text { effective }\end{array}$ & $\begin{array}{l}\text { None } \\
\text { stated }\end{array}$ & $\begin{array}{l}\text { Not } \\
\text { stated }\end{array}$ \\
\hline
\end{tabular}

AUF Agence Universitaire de la Francophonie, B\&MGF Bill \&Melinda Gates, DALYs Disability-adjusted-life-years, $N C I$ National Cancer Institute, $Q A L Y S$ quality-adjusted-life-years, YLS years of life save 


\section{Risk of bias (RoB) within the selected studies}

Table 2 below summarizes the risk of bias assessment using the CHEC checklist as proposed by Wijnen et al., (2016) .

\section{Table 3: Risk of bias in the selected studies}

\begin{tabular}{|c|c|c|c|c|c|c|c|c|c|c|c|c|c|}
\hline & CHEC items & $\begin{array}{l}\text { Andres- } \\
\text { Gamboa et } \\
\text { al. (34) }\end{array}$ & $\begin{array}{c}\text { Camp } \\
\text { os et } \\
\text { al., } \\
(\mathbf{3 5})\end{array}$ & $\begin{array}{l}\text { Chantha } \\
\text { vilay et } \\
\text { al. (28) }\end{array}$ & $\begin{array}{c}\text { Ezat and } \\
\text { Aljunid } \\
\text { (8) }\end{array}$ & $\begin{array}{c}\text { Khatib } \\
\text { i et al. } \\
\text { (30) }\end{array}$ & $\begin{array}{l}\text { Levi } \\
\text { n et } \\
\text { al. } \\
(36)\end{array}$ & $\begin{array}{l}\text { Molina } \\
\text { et al. } \\
\text { (33) }\end{array}$ & $\begin{array}{c}\text { Prinja } \\
\text { et al. } \\
\text { (32) }\end{array}$ & $\begin{array}{l}\text { Sharm } \\
\text { a et al. } \\
\text { (26) }\end{array}$ & $\begin{array}{l}\text { Sinano } \\
\text { vic et } \\
\text { al. (27) }\end{array}$ & $\begin{array}{l}\text { Termrung } \\
\text { ruanglert } \\
\text { et al. (31) }\end{array}$ & $\begin{array}{l}\text { Vanni et } \\
\text { al. (29) }\end{array}$ \\
\hline 1 & Clearly defined population & Yes & Yes & Yes & Yes & Yes & Yes & Yes & Yes & Yes & Yes & Yes & Yes \\
\hline 2 & Clearly defined comparator & Yes & Yes & Yes & Yes & Yes & Yes & Yes & Yes & Yes & Yes & Yes & Yes \\
\hline 3 & Research question & Yes & Yes & Yes & Yes & Yes & Yes & Yes & Yes & Yes & Yes & Yes & Yes \\
\hline 4 & Appropriate design & Yes & Yes & Yes & Yes & Yes & Yes & Yes & Yes & Yes & Yes & Yes & Yes \\
\hline 5 & Valid assumptions & Yes & Yes & Yes & Yes & Yes & Yes & Yes & Yes & Yes & Yes & Yes & Yes \\
\hline 6 & Appropriate time horizon & Yes & Yes & Yes & Yes & Yes & Yes & Yes & Yes & Yes & Yes & Yes & Yes \\
\hline 7 & Perspective chosen & Yes & Yes & Yes & Yes & Yes & Yes & Yes & Yes & Yes & Yes & Yes & Yes \\
\hline 8 & All costs included & Yes & Yes & Yes & Yes & Yes & Yes & Yes & Yes & Yes & Yes & Yes & Yes \\
\hline 9 & Appropriate cost measures & Yes & Yes & Yes & Yes & Yes & Yes & Yes & Yes & Yes & Yes & Yes & Yes \\
\hline 10 & Appropriate cost valuation & Yes & Yes & Yes & Yes & Yes & Yes & Yes & Yes & Yes & Yes & Yes & Yes \\
\hline 11 & All outcomes identified & Yes & Yes & Yes & Yes & Yes & Yes & Yes & Yes & Yes & Yes & Yes & Yes \\
\hline 12 & Measured all outcomes & Yes & Yes & Yes & Yes & Yes & Yes & Yes & Yes & Yes & Yes & Yes & Yes \\
\hline 13 & Outcomes valued correctly & Yes & Yes & Yes & Yes & Yes & Yes & Yes & Yes & Yes & Yes & Yes & Yes \\
\hline 14 & ICERs/ ICURs calculated & Yes & Yes & Yes & Yes & Yes & Yes & Yes & Yes & Yes & Yes & Yes & Yes \\
\hline 15 & Discounted costs and benefits & Yes & Yes & Yes & Yes & Yes & Yes & Yes & Yes & Yes & Yes & Yes & Yes \\
\hline 16 & Sensitivity analysis & Yes & Yes & Yes & Yes & Yes & Yes & Yes & Yes & Yes & Yes & Yes & Yes \\
\hline 17 & Conclusions linked to results & Yes & Yes & Yes & Yes & Yes & Yes & Yes & Yes & Yes & Yes & Yes & Yes \\
\hline 18 & Generalizability discussed & No & No & No & No & No & No & No & No & No & No & No & No \\
\hline 19 & Competing interests declared & No & Yes & Yes & No & No & Yes & Yes & No & No & Yes & Yes & No \\
\hline 20 & Funding disclosed & No & Yes & Yes & No & Yes & Yes & Yes & No & Yes & Yes & No & No \\
\hline
\end{tabular}




\section{Results synthesis}

There were significant differences across the selected studies, hence, this review focussed on describing the results, and their application rather than undertaking a metaanalysis of key findings. The study population for both screening and vaccination interventions was predominantly female while only one study included males (29). The latter findings revealed that including males makes HPV vaccination intervention non costeffective (29).

\section{HPV vaccination}

Eleven studies reported vaccinating girls aged 9-13 years was highly cost-effective; however, one study found otherwise but assumed a low coverage and a very high cost of vaccines (30). Regarding the number of doses required to achieve lifelong immunity against HPV, four studies assessed the effectiveness of vaccination with three doses $(27,28,30,33)$. Two more studies assessed the use of two doses of vaccination $(29,32)$ while another two studies focused on single dose vaccinations $(26,31)$. Determining the number of vaccine doses that produced the best cost-effectiveness results was beyond the scope of this review.

Furthermore, the vaccine efficacy significantly influences cost-effectiveness outcomes for vaccination programmes $(26,31,32)$. The findings suggest that HPV vaccination interventions have potential cost-effectiveness in LMICs. Vaccination without any other intervention appears to be very cost effective (ICER I\$3.19-821.85/ QALY/ DALY) provided the coverage is maintained between $70-100 \%$ based on the reviewed evidence $(32,33)$. However, an Iranian-based study determined that three dose vaccination was not costeffective (I\$15608.94/QALY) (30). 


\section{HPV Screening}

The reported age range for screening was 21-69 years (34), 35 years (35), and 35-45 years (36). Notably, two of the included studies reported initiating screening at an early age (below 30 years) improved cost-effectiveness $(28,34)$. The screening approaches also varied across studies and included VIA (26-28,35), HPV DNA testing (26-28,34-36), and cytological analysis or Pap smear $(8,26-29,34,36)$. Cost-effectiveness varied significantly when drawing comparisons based on the screening approaches used.

The number of visits required to complete a screening test is important because the associated loss-to-follow-up (LTFU) undermines the overall effectiveness of screening interventions. HPV DNA analysis appears to be less cost-effective whenever the LTFU exceeds $40 \%$ of the primary population $(28,34)$. Notably, reducing the uptake of follow-up services may contribute to a higher mortality rate in comparison with low coverage with respect to screening interventions.

The reviewed evidence shows that screening interventions may not be cost-effective at lower coverage but are cost-effective at $50 \%$ coverage and become very attractive options at a coverage of $70 \%$ of the population at risk. In addition, the findings reveal that organized cervical cancer prevention programmes achieve a higher coverage of the population at risk than opportunistic programmes and result in better cost-effectiveness outcomes than opportunistic interventions $(28,34)$.

The cost range for VIA screening was I\$3.65-I\$55.81 whereas HPV DNA testing and cytological analysis across costs ranged between I\$9.0-I\$229.93 and I\$3.74-I\$69.20 respectively. The ICERs reported in different studies varied widely with two studies reporting that screening with no other intervention was cost-effective $(34,35)$. The implementation of two visit HPV DNA testing appears to be very cost-effective recording an ICER of 
I $\$ 105.21 /$ YLS (36). When the loss-to-follow-up is below 40\%, HPV DNA testing appears to yield higher cost-effectiveness when compared with other methods (35). Otherwise, there is a $73 \%$ probability that VIA will be more cost-effective screening strategy in comparison with HPV DNA testing when implemented (28). 
Table 4: Summary of individual study results

\begin{tabular}{|c|c|c|c|c|c|c|c|c|c|c|}
\hline & Intervention & $\begin{array}{l}\text { Frequency/ } \\
\text { Doses }\end{array}$ & Coverage & $\begin{array}{l}\text { Efficacy/ } \\
\text { sensitivity }\end{array}$ & $\begin{array}{l}\text { Reference } \\
\text { year }\end{array}$ & $\begin{array}{l}\text { Cost } \\
\text { (Original) }\end{array}$ & ICER (Original) & $\begin{array}{l}\text { Discounted } \\
\text { costs (I\$) }\end{array}$ & $\begin{array}{l}\text { Discounted } \\
\text { ICERs } \\
\text { (I\$/) }\end{array}$ & Remarks \\
\hline \multicolumn{11}{|c|}{ SCREENING ONLY } \\
\hline \multirow[t]{2}{*}{$\begin{array}{l}\text { Andres-Gamboa et } \\
\text { al. (34) }\end{array}$} & Cytology & $3 \mathrm{x}$ & $50-100 \%$ & $\begin{array}{l}72.7(50- \\
81.5) \%\end{array}$ & 2007 & $\begin{array}{l}6.34(5.9- \\
12.67)\end{array}$ & & 4.72 & & \\
\hline & HPV DNA & $2 \mathrm{x}$ & $50-100 \%$ & $\begin{array}{l}90(86.4- \\
93.7) \%\end{array}$ & & $\begin{array}{l}\$ 12.10(8.30- \\
62.53)\end{array}$ & USD\$44/YLS & 9.00 & 32.74 & $\begin{array}{l}\text { Very } \\
\text { cost- } \\
\text { effective }\end{array}$ \\
\hline \multirow[t]{2}{*}{ Campos et al. (35) } & VIA & $1 \mathrm{x}$ & $30-100 \%$ & $40-60 \%$ & 2005 & & & & & \\
\hline & HPV DNA & $2 \mathrm{x}$ & $30-100 \%$ & $70-90 \%$ & & $\begin{array}{l}\$ 17.06- \\
19.07\end{array}$ & I\$300/YLS & $\begin{array}{l}11.97- \\
13.38\end{array}$ & 210.41 & $\begin{array}{l}\text { Cost- } \\
\text { effective }\end{array}$ \\
\hline \multirow[t]{2}{*}{ Levin et al. (36) } & HPV DNA & $2 \mathrm{x}$ & & $\begin{array}{l}89.7(50- \\
100) \%\end{array}$ & 2005 & $\$ 45.89$ & \$80-150/YLS & 32.19 & 105.21 & $\begin{array}{l}\text { Very } \\
\text { cost- } \\
\text { effective }\end{array}$ \\
\hline & Cytology & & & $\begin{array}{l}63(50- \\
100) \% \\
\end{array}$ & & $\$ 5.33$ & & 3.74 & & \\
\hline \multicolumn{11}{|c|}{ VACCINATION ONLY } \\
\hline Khatibi et al. (30) & Vaccination & $3 x$ & $60 \%$ & $100 \%$ & 2013 & IRR4181805 & $\begin{array}{l}\text { IRR439000000 251000000- } \\
842000000 / \text { QALY }\end{array}$ & 148.38 & 15608.94 & $\begin{array}{l}\text { Not cost- } \\
\text { effective }\end{array}$ \\
\hline $\begin{array}{l}\text { Termrungruanglert } \\
\text { et al. (31) }\end{array}$ & Vaccination & $1 \mathrm{x}$ & $80-100 \%$ & $90-99 \%$ & 2009 & $\begin{array}{l}6,189- \\
12,378 \text { bhat }\end{array}$ & $\begin{array}{l}\text { 160,649-406394 } \\
\text { baht/QALY }\end{array}$ & $\begin{array}{l}146.67- \\
293.34\end{array}$ & $\begin{array}{l}3807.32- \\
9630.79 \\
\end{array}$ & $\begin{array}{l}\text { Cost- } \\
\text { effective }\end{array}$ \\
\hline Molina et al. (33) & Vaccination & $3 \mathrm{x}$ & $85-100 \%$ & $\begin{array}{l}48.4- \\
96.8 \%\end{array}$ & 2013 & $\begin{array}{l}\$ 13.10- \\
13.79\end{array}$ & \$843-925/DALY & $\begin{array}{l}11.64- \\
12.25\end{array}$ & $\begin{array}{l}749.00- \\
821.85\end{array}$ & $\begin{array}{l}\text { Very } \\
\text { cost- } \\
\text { effective }\end{array}$ \\
\hline Prinja et al. (32) & Vaccination & $2 \mathrm{x}$ & $70 \%$ & $93 \%$ & 2015 & & I\$3.38/DALY & 3.19 & 3.19 & $\begin{array}{l}\text { Very } \\
\text { cost- } \\
\text { effective }\end{array}$ \\
\hline \multicolumn{11}{|c|}{ VACCINATION AND SCREENING } \\
\hline \multirow[t]{2}{*}{ Sharma et al. (26) } & $\begin{array}{l}\text { Vaccination } \\
+\end{array}$ & $1 \mathrm{x}$ & $80 \%$ & & 2005 & I\$10-100 & I\$2260- I\$3550/YLS & $7.01-70.14$ & 2980.86 & $\begin{array}{l}\text { Cost- } \\
\text { effective }\end{array}$ \\
\hline & VIA & $1 \mathrm{x}$ & & $75-100 \%$ & & I $\$ 5.21$ & & 3.65 & & $\begin{array}{l}\text { Cost- } \\
\text { effective }\end{array}$ \\
\hline
\end{tabular}




\begin{tabular}{|c|c|c|c|c|c|c|c|c|c|c|}
\hline & HPV DNA & $2 \mathrm{x}$ & $>60 \%$ & $40-60 \%$ & & I\$26.11 & I\$4250/YLS & 18.31 & & $\begin{array}{l}\text { Very } \\
\text { cost- } \\
\text { effective }\end{array}$ \\
\hline & Cytology & $3 x$ & & & & I\$18.92 & & 13.27 & & $\begin{array}{l}\text { Not cost- } \\
\text { effective }\end{array}$ \\
\hline \multirow[t]{4}{*}{$\begin{array}{l}\text { Sinanovic et al. } \\
\text { (27) }\end{array}$} & Vaccination & $3 \mathrm{x}$ & $80 \%$ & $90 \%$ & 2007 & $\$ 281-289$ & \$1078-1460/QALY & $\begin{array}{l}209.09- \\
215.04\end{array}$ & $\begin{array}{l}802.13- \\
1086.38 \\
\end{array}$ & $\begin{array}{l}\text { Cost- } \\
\text { effective }\end{array}$ \\
\hline & VIA & & & & & $\$ 75$ & & 55.81 & & \\
\hline & HPV DNA & & & & & $\$ 309$ & & 229.93 & & \\
\hline & Cytology & & & & & $\$ 93$ & & 69.20 & & \\
\hline \multirow[t]{4}{*}{$\begin{array}{l}\text { Chanthavilay et al. } \\
\text { (28) }\end{array}$} & Vaccination & $3 \mathrm{x}$ & $\begin{array}{l}70(30- \\
80)\end{array}$ & Lifelong & 2013 & I $\$ 33.5$ & I\$2544/DALY & 29.76 & 2260.31 & $\begin{array}{l}\text { Very } \\
\text { cost- } \\
\text { effective }\end{array}$ \\
\hline & VIA or & $1 \mathrm{x}$ & $\begin{array}{l}50(10- \\
80) \%\end{array}$ & $\begin{array}{l}73.2(66.5- \\
80.0 \%)\end{array}$ & & I\$ $\$ 26.45$ & I\$351-2544/DALY & 23.50 & $\begin{array}{l}311.86- \\
2260.31\end{array}$ & $\begin{array}{l}\text { Very } \\
\text { cost- } \\
\text { effective }\end{array}$ \\
\hline & HPV DNA & $2 \mathrm{x}$ & $\begin{array}{l}50(10- \\
80) \%\end{array}$ & $\begin{array}{l}81.5(76.5- \\
85.8 \%) \\
\end{array}$ & & I $\$ 47.18$ & I\$2102-4391/DALY & 41.92 & $\begin{array}{l}1867.6- \\
3901.35 \\
\end{array}$ & $\begin{array}{l}\text { Cost- } \\
\text { effective }\end{array}$ \\
\hline & Cytology & $3 x$ & $\begin{array}{l}50(10- \\
80) \%\end{array}$ & $\begin{array}{l}59(29- \\
82 \%)\end{array}$ & & I $\$ 48.27$ & I\$3455/DALY & 42.89 & 3069.72 & $\begin{array}{l}\text { Cost- } \\
\text { effective }\end{array}$ \\
\hline \multirow[t]{2}{*}{$\begin{array}{l}\text { Ezat and Aljunid } \\
\text { (8) }\end{array}$} & Vaccination & & & & 2005 & RM300-700 & RM35347/QALY & $\begin{array}{l}60.98- \\
142.31\end{array}$ & 7185.64 & $\begin{array}{l}\text { Cost- } \\
\text { effective }\end{array}$ \\
\hline & Cytology & & $\begin{array}{l}70(40- \\
90) \%\end{array}$ & & & RM41-72 & RM947/QALY & $8.33-14.64$ & 192.53 & $\begin{array}{l}\text { Very } \\
\text { cost- } \\
\text { effective }\end{array}$ \\
\hline \multirow[t]{2}{*}{ Vanni et al. (29) } & Vaccination & $2 \mathrm{x}$ & $50-90 \%$ & Lifelong & 2008 & $\$ 30-676$ & \$13576/QALY & $\begin{array}{l}19.34- \\
435.76\end{array}$ & 8751.21 & $\begin{array}{l}\text { Very } \\
\text { cost- } \\
\text { effective }\end{array}$ \\
\hline & Cytology & & $63 \%$ & $58 \%$ & & & & & & \\
\hline
\end{tabular}

$D N A$ deoxyribonucleic acid, $H P V$ human papilloma virus, ICER incremental cost-effectiveness ratio, ICUR incremental cost-utility ratio, DALYS Disabilityadjusted-life-years, NCI National Cancer Institute, $Q A L Y s$ quality-adjusted-life-years, VIA visual inspection with acetic acid, YLS years of life saved 


\section{HPV vaccination combined with screening}

Combining HPV vaccination with any of the screening approaches potentially results in better cost-effectiveness outcomes as reported in four studies when compared with either screening only or vaccination only interventions $(8,28,29)$. However, vaccination appears to be cost-effective only when combined with either VIA or HPV DNA testing but not in combination with cytology (ICER: I\$2980.86/ YLS (26).

Vaccination combined with VIA screening was associated with the highest (85.70\%) decrease in cervical carcinoma cases (28) whereas two-times HPV DNA testing recorded the lowest $(26-50 \%)$ reduction in cancer cases. When ranked based on the incremental costeffectiveness ratios, there is an overlap of the ICERs across the three interventions namely screening only (ICERs I\$37.4-I\$210.41/YLS), vaccination combined with screening (ICERs I\$192.53-I\$8751.21 per QALY/ DALY/ YLS), and vaccination-only interventions (ICERs I\$3.19-I\$15608.94/QALY/ DALY).

The findings suggest that combining VIA screening and HPV vaccination could be the most cost-effective approach in reducing the lifetime risk for cervical neoplasms linked to HPV by $85.7 \%$ (28). Regarding the approach that could be most cost-efficacious between screening interventions and vaccination programmes, probabilistic sensitivity analyses of cost-effectiveness results revealed that five yearly screening had a 70-80\% probability for better cost-effectiveness in comparison with HPV vaccination programmes (28). 


\section{Discussion}

The results from this review suggest HPV vaccination and screening interventions have potential cost-effectiveness in decreasing the lifetime risk as well as mortality (26$85.7 \%$ ) associated with cervical carcinoma. The interventions are useful in mitigating the huge disease and economic burden caused by HPV infections. Prevention approaches namely screening, vaccination, or vaccination combined with screening are potentially cost-effective. The interventions become even more cost-effective when screening and vaccination programmes are combined. The findings are in tandem with a previous review, which reported vaccination only programmes are cost-effective even in settings that lack organized screening interventions (15).

Based on the outcomes of sensitivity analyses conducted across the studies, the findings revealed a host of factors that significantly influence the cost effectiveness of either screening or HPV vaccination interventions. Key among these factors is the age for delivering the vaccination or screening programmes as well as the number of doses or visits required for vaccination and screening respectively. With regard to vaccination age, the evidence suggests better outcomes with interventions delivered between the age of nine and fifteen years. However, there was insufficient evidence to conclude whether the different dose schedules influence the cost-effectiveness outcomes for HPV vaccination. Regarding screening interventions, two studies revealed that early screening from twenty years is more cost-effective in comparison with screening from thirty years and above $(28,34)$.

The reviewed evidence also suggests that high coverage for both screening and HPV vaccination interventions are critical factors that influence cost-effectiveness outcomes. The interventions appear to be less cost-effective at a coverage below $50 \%$ of the population at risk. Regarding the potential impact of test sensitivity on HPV screening outcomes, the 
findings are inconclusive due to contradictory results from the included studies. One study concluded that the sensitivity of the test used has negligible impact on the cost-effectiveness outcomes for screening interventions (35) while another contradicted these findings (28). The remainder of the studies did not conclude on the impact of the screening test sensitivity on the cost-effectiveness results.

\section{Policy and practice implications}

The findings of this review suggested that implementing organized programmes as opposed to opportunistic interventions potentially yields better cost-effectiveness outcomes due to improved coverage $(28,34)$. This is consistent with the WHO recommendations emphasizing on the need for organized screening and vaccination interventions for better outcomes (40). Similarly, evidence from high-income countries demonstrates the importance for organized programmes (41).

The cost-effectiveness of screening and HPV vaccination interventions also depends on the screening method used, number of visits required, screening age, coverage, test sensitivity and specificity, as well as any follow-up requirements. Generally, any of the three screening methods (VIA, HPV DNA testing, and cytological testing or Pap smear) are costeffective as a stand-alone intervention. However, combining screening interventions with a vaccination programme yields superior cost-effectiveness outcomes. Comparatively, VIA seems to be the most cost-effective screening strategy whereas the cytological testing seems to be least cost-effective when implemented alone or in combination with HPV vaccination. One of the factors that makes VIA significantly more cost-effective than other screening methods is its reduced loss-to-follow-up (LTFU) $(28,35)$.

LTFU is of great importance especially in cases where there are confirmed positives following a screening test who do not turn-up for further testing and treatment (34). This also 
applies to HPV vaccination interventions that require women to return to the facility for booster doses before attaining full immunity. LTFU increases the mortality rates therefore making a particular screening test less cost-effective. Therefore, public health practitioners should take into account the potential LTFU when choosing the most suitable screening method. In addition, there is need for relevant policy guidelines aimed at reducing the high LTFU witnessed in LMICs by opting for screening and HPV vaccination interventions that do not require women to frequent the health facility.

The success of a VIA screening intervention depends on the context and may suffer some setbacks caused by cultural barriers that undermine women's adherence (8). Notably, decision makers should have a clear understanding of the contextual factors such as women's preferences regarding the available screening methods. It may be prudent to opt for other culturally acceptable interventions that do not discourage women from taking up screening such as HPV DNA testing through a self-collection of HPV samples approach (35). Such an approach may be more costly when compared with sample collection by healthcare providers but useful in overcoming certain cultural barriers to cervical screening $(35,42,43)$.

The reviewed evidence also suggests that providing screening at an early age of twenty (20) years has a higher cost-effectiveness and better outcomes in comparison with screening women after they attain 30 years (28),(34). This is important considering the fact that most screening interventions target women after attaining 30 years despite the evidence showing a peak incidence of HPV infection below this age $(6,34)$.

Similarly, age influences the cost-effectiveness of immunization programmes targeting HPV eradication. Consistent with current evidence (44), this SR affirms that immunizing girls at an early age (9-13 years) yields higher benefits in LMICs (26,28,31-33). However, one of the reviewed studies established that providing HPV vaccination to girls 
aged 15 years was not a cost-effective option (30). Notably, the latter study lacked country data in model calibration, assumed a low coverage and had the highest cost estimates in comparison with other studies included in this review. There was also no evidence with regard to the potential cost-effectiveness of HPV vaccination in older women.

Generally, screening and HPV vaccination programmes with higher coverage tend to yield better cost-effectiveness outcomes. This is evidenced by the sensitivity analyses conducted across the selected studies revealing coverage as a critical factor in costeffectiveness of the interventions $(8,31,34)$.

One of the challenges identified in LMICs is the low coverage of cervical cancer prevention interventions (45). The interventions only surpassed the cot-effectiveness threshold at higher coverage above 50-70\% (28) but this remains an uphill task considering the fragile healthcare system in LMICs. As discussed above, increased coverage is achievable through the implementation of organised interventions. A case example is Rwanda's vaccination campaign that attained a $93.23 \%$ coverage surpassing the recommended threshold for immunization programmes (46). Considering the evidence that HPV vaccination and screening have potential cost-effectiveness even as stand-alone interventions, progressively increasing coverage through organised programmes can significantly reduce the risk for cancer and associated deaths.

The unit cost of vaccines is another key determinant influencing cost-effectiveness outcomes for HPV vaccination interventions in LMICs (27,32). For instance, doubling the unit price of HPV vaccine in Thailand would make the programme non cost-effective (31). Higher costs may limit the coverage of vaccination interventions within the context of LMICs. This may also translate to an increase in taxation as well as a rise in out-of-pocket expenditure to cover for the additional costs. This further worsens the economic burden of 
cervical carcinoma associated with HPV infections and perpetrates the cycle of poverty in impoverished societies.

\section{Implications for research}

Additional studies are essential in ascertaining whether including males in HPV vaccination interventions influences the cost-effectiveness outcomes in LMICs. The evidence reviewed was not conclusive on this aspect considering that most included studies never incorporated the potential effect of including males during the model calibration process. One study reported that vaccinating males is a less attractive option (29) whereas another study argued that vaccinating males has no usefulness in settings that have 100\% HPV vaccination coverage amongst females (31). This contradicts available evidence, which demonstrates that inclusion of males is a highly beneficial strategy in decreasing the lifetime risk of HPV associated infections $(41,47)$, hence worth further investigating.

There was no sufficient evidence to conclude whether the number of doses required to complete a vaccination programme against HPV influences the cost-effectiveness of vaccination interventions. Across all the studies, subjects acquired lifelong immunity upon completion of the immunization schedule regardless of the number of doses. It is important to note the uncertainty in lifelong immunity considering the fact that there are no lifetime studies conducted to that effect considering the recent rollout of HPV vaccination programmes in 2007. Based on immunization guidelines from the CDC and the WHO, the doses required to achieve lifelong immunity are dependent upon the type of vaccine used and the age of vaccination $(44,48)$.

\section{Limitations}


This review has a number of limitations that would significantly influence the generalizability of the findings. The studies included in the final synthesis varied significantly in terms of their contexts, perspective, population, costing approaches, and measurement of effects employed by the researcher. Most of the included studies were devoid of countrybased data on the age-specific incidence of HPV infections making it impossible to develop an accurate model for HPV's natural history and cervical carcinogenesis.

Lastly, only one of the included studies used a dynamic model and accounted for the potential influence of herd immunity on the cost-effectiveness outcomes. Considering the high coverage of vaccination intervention assessed by the included studies, it would be prudent to assess the influence that herd immunity exerts on the cost-effectiveness outcomes. It is possible that the effects of the interventions that relied on the government perspective were overestimated because they did not take into account the costs associated with access to services as well as women's waiting times.

\section{Conclusions}

This SR synthesized the existing evidence with regard to the potential costeffectiveness of cervical screening and HPV vaccination within the context of LMICs. The reviewed evidence suggests that HPV vaccination and screening interventions may be costeffective in reducing the lifetime risk, economic burden, and mortality caused by HPV linked cervical carcinoma. In addition, combining HPV vaccination with VIA screening has potential for better cost-effectiveness outcomes within the context of LMICs in comparison with either screening or vaccination only programmes. The cost-effectiveness outcomes of HPV vaccination and screening interventions are dependent on age, screening method used, intervention coverage, and the number of doses or visits required for vaccination and screening respectively. There is need for additional studies on the duration of protection 
achieved by the different dose schedules of HPV vaccines as well as the inclusion of boys in the vaccination programme.

\section{Data availability}

The datasets generated during and/or analysed during the current study are available from the corresponding author on reasonable request through bernardokeah@gmail.com or c.h.ridyard@bangor.ac.uk.

\section{Authors contributions}

B.O. conceived the idea, designed the study protocol and was the first reviewer. C.R. verified the study protocol, was the second reviewer and supervised the study. The authors discussed the findings of this study and contributed to the final report.

\section{Funding}

None

\section{Conflict of interest}

No potential conflicts of interest 


\section{References}

1. WHO. Cervical cancer. WHO [Internet]. 2018 [cited 2019 Jul 17]; Available from: https://www.who.int/cancer/prevention/diagnosis-screening/cervical-cancer/en/

2. Bray F, Ferlay J, Soerjomataram I, Siegel RL, Torre LA, Jemal A. Global cancer statistics 2018: GLOBOCAN estimates of incidence and mortality worldwide for 36 cancers in 185 countries. CA Cancer J Clin [Internet]. 2018 Nov [cited 2019 Jul 19];68(6):394-424. Available from: http://doi.wiley.com/10.3322/caac.21492

3. Cohen P, Jhingran A, Oaknin A. Cervical cancer. Lancet [Internet]. 2019 [cited 2019 Jul 21];393(10167):169-82. Available from: https://www.sciencedirect.com/science/article/pii/S014067361832470X

4. Cheikh A, Majjaoui S El, Ismaili N, Cheikh Z, Bouajaj J, Nejjari C, et al. Evaluation of the cost of cervical cancer at the national institute of oncology, Rabat. Pan Afr Med J [Internet]. 2016 [cited 2019 Aug 29];23. Available from: https://www.ajol.info/index.php/pamj/article/view/139545

5. Ginsburg O, Bray F, Coleman M., Vanderpuye V, Eniu A, Kotha S., et al. The global burden of women's cancers: A grand challenge in global health. Lancet [Internet]. 2017 [cited 2019 Jul 19];no pagination. Available from: https://www.sciencedirect.com/science/article/pii/S0140673616313927

6. de Martel C, Plummer M, Vignat J, Franceschi S. Worldwide burden of cancer attributable to HPV by site, country and HPV type. Int J Cancer [Internet]. 2017 [cited 2019 Jul 19];141(4):664-70. Available from: https://onlinelibrary.wiley.com/doi/abs/10.1002/ijc.30716\%4010.1002/\%28ISSN\%291 097-0215.world_cancer_congress2018 
7. Smittenaar CR, Petersen KA, Stewart K, Moitt N. Cancer incidence and mortality projections in the UK until 2035. Br J Cancer [Internet]. 2016 [cited 2019 Jul 19];115(9):1147-55. Available from: https://www.nature.com/articles/bjc2016304

8. Ezat WPS, Aljunid S. Cost-Effectiveness of HPV Vaccination in the Prevention of Cervical Cancer in Malaysia. ASIAN PACIFIC J CANCER Prev [Internet]. 2010;11(1):79-90. Available from: https://www.researchgate.net/profile/Sharifa_Ezat_Ezat/publication/44900594_CostEffectiveness_of_HPV_Vaccination_in_the_Prevention_of_Cervical_Cancer_in_Mala ysia/links/00b4952670f833a7fe000000.pdf

9. Parkin DM, Hämmerl L, Ferlay J, Kantelhardt EJ. Cancer in Africa 2018: the role of infections. Int J Cancer [Internet]. 2019 Jun 29 [cited 2019 Jul 19]; Available from: http://doi.wiley.com/10.1002/ijc.32538

10. International Agency for Research on Cancer. Cancer Today [Internet]. 2018 [cited 2019 Jul 19]. Available from: https://gco.iarc.fr/today/home

11. Carneiro SR, Fagundes M de A, do Rosário P de JO, Neves LMT, Souza G da S, Pinheiro M da CN. Five-year survival and associated factors in women treated for cervical cancer at a reference hospital in the Brazilian Amazon. Tornesello ML, editor. PLoS One [Internet]. 2017 Nov 16 [cited 2019 Jul 21];12(11):e0187579. Available from: http://dx.plos.org/10.1371/journal.pone.0187579

12. WHO. Comprehensive cervical cancer control A guide to essential practice [Internet]. 2nd ed. Geneva: WHO Press; 2014. 364 p. Available from: https://www.who.int/reproductivehealth/publications/cancers/cervical-cancer-guide/en/

13. Jindal HA, Kaur A, Murugan S. Human papilloma virus vaccine for low and middle income countries: A step too soon? [Internet]. Vol. 13, Human Vaccines and 
Immunotherapeutics. 2017 [cited 2019 Jul 17]. p. 2723-5. Available from: https://www.tandfonline.com/doi/full/10.1080/21645515.2017.1358837

14. Mirghani H, Jung A., Fakhry C. Primary, secondary and tertiary prevention of human papillomavirus-driven head and neck cancers. Eur J Cancer [Internet]. 2017 [cited 2019 Aug 16];78:105-15. Available from:

https://www.sciencedirect.com/science/article/pii/S0959804917308390

15. Fesenfeld M, Hutubessy R, Jit M. Cost-effectiveness of human papillomavirus vaccination in low and middle income countries: a systematic review. Vaccine [Internet]. 2013 Aug [cited 2019 Aug 8];31(37):3786-804. Available from: https://www.sciencedirect.com/science/article/pii/S0264410X13008529

16. Ekwunife OI, Grote AG, Mosch C, O’Mahony JF, Lhachimi SK. Assessing costeffectiveness of HPV vaccines with decision analytic models: what are the distinct challenges of low- and middle-income countries? A protocol for a systematic review. Syst Rev [Internet]. 2015 Dec 12 [cited 2019 Jul 26];4(1):68. Available from: https://systematicreviewsjournal.biomedcentral.com/articles/10.1186/s13643-015$0057-8$

17. Moher D, Shamseer L, Clarke M, Ghersi D, Liberati A, Petticrew M, et al. Preferred reporting items for systematic review and meta-analysis protocols (PRISMA-P) 2015 statement. Syst Rev [Internet]. 2015 Dec 1 [cited 2019 Jul 24];4(1):1. Available from: https://systematicreviewsjournal.biomedcentral.com/articles/10.1186/2046-4053-4-1

18. Lockwood C, Oh EG. Systematic reviews: Guidelines, tools and checklists for authors. Vol. 19, Nursing and Health Sciences. John Wiley \& Sons, Ltd (10.1111); 2017. p. $273-7$.

19. Hopewell S, McDonald S, Clarke MJ, Egger M. Grey literature in meta-analyses of 
randomized trials of health care interventions. Cochrane Database Syst Rev [Internet]. 2007 Apr 18 [cited 2019 Jul 17]; Available from:

http://doi.wiley.com/10.1002/14651858.MR000010.pub3

20. Wijnen B, Van Mastrigt G, Redekop W, Majoie H, De Kinderen R, Evers SMAA. How to prepare a systematic review of economic evaluations for informing evidencebased healthcare decisions: data extraction, risk of bias, and transferability (part 3/3) [Internet]. Vol. 16, Expert Review of Pharmacoeconomics and Outcomes Research. 2016 [cited 2019 Jul 17]. p. 723-32. Available from: https://www.tandfonline.com/doi/full/10.1080/14737167.2016.1246961

21. van Mastrigt G, Hiligsmann M, Arts JJC, Broos P, Kleijnen J, Evers S, et al. How to prepare a systematic review of economic evaluations for informing evidence-based healthcare decisions: a five-step approach (part 1/3). Expert Rev Pharmacoecon Outcomes Res [Internet]. 2016 Nov 2 [cited 2019 Jul 24];16(6):689-704. Available from: https://www.tandfonline.com/doi/full/10.1080/14737167.2016.1246960

22. Drummond M, Jefferson TO. Guidelines for authors and peer reviewers of economic. Br Med J [Internet]. 1996 [cited 2019 Jul 17];313(August):275-83. Available from: https://www.bmj.com/content/313/7052/275.pdf+html

23. Fukuda H, Imanaka Y. Assessment of transparency of cost estimates in economic evaluations of patient safety programmes. J Eval Clin Pract [Internet]. 2009 Jun [cited 2019 Jul 23];15(3):451-9. Available from: http://doi.wiley.com/10.1111/j.13652753.2008.01033.x

24. Dakin H, Devlin N, Feng Y, Rice N, O’Neill P, Parkin D. The Influence of CostEffectiveness and Other Factors on Nice Decisions. Health Econ [Internet]. 2015 Oct [cited 2019 Jul 17];24(10):1256-71. Available from: 
http://doi.wiley.com/10.1002/hec.3086

25. WHO | Purchasing Power Parity 2005 [Internet]. [cited 2020 Mar 9]. Available from: https://www.who.int/choice/costs/ppp/en/

26. Sharma M, Ortendahl J, van der Ham E, Sy S, Kim JJ. Cost-effectiveness of human papillomavirus vaccination and cervical cancer screening in Thailand. BJOG-AN Int J Obstet Gynaecol [Internet]. 2012 Jan;119(2):166-76. Available from: https://obgyn.onlinelibrary.wiley.com/doi/full/10.1111/j.1471-0528.2011.02974.x

27. Sinanovic E, Moodley J, Barone MA, Mall S, Cleary S, Harries J. The potential costeffectiveness of adding a human papillomavirus vaccine to the cervical cancer screening programme in South Africa. Vaccine [Internet]. 2009 Oct;27(44):6196-202. Available from:

https://www.sciencedirect.com/science/article/pii/S0264410X09011670

28. Chanthavilay P, Reinharz D, Mayxay M, Phongsavan K, Marsden DE, Moore L, et al. Economic Evaluation of Screening Strategies Combined with HPV Vaccination of Preadolescent Girls for the Prevention of Cervical Cancer in Vientiane, Lao PDR. PLoS One [Internet]. 2016 Sep;11(9). Available from: https://journals.plos.org/plosone/article?id=10.1371/journal.pone.0162915

29. Vanni T, Luz PM, Foss A, Mesa-Frias M, Legood R. Economic modelling assessment of the HPV quadrivalent vaccine in Brazil: A dynamic individual-based approach. Vaccine [Internet]. 2012 Jul;30(32):4866-71. Available from: https://www.sciencedirect.com/science/article/pii/S0264410X12006330

30. Khatibi M, Rasekh HR, Shahverdi Z, Jamshidi HR. Cost-Effectiveness Evaluation of Quadrivalent Human Papilloma Virus Vaccine for HPV-Related Disease in Iran. Iran J Pharm Res [Internet]. 2014;13(S):225-34. Available from: 
https://www.ncbi.nlm.nih.gov/pmc/articles/PMC3977074/

31. Termrungruanglert W, Havanond P, Khemapech N, Lertmaharit S, Pongpanich S, Khorprasert C, et al. Cost and effectiveness evaluation of prophylactic HPV vaccine in developing countries. Value Health [Internet]. 2012;15(1 Suppl):S29-34. Available from: https://www.sciencedirect.com/science/article/pii/S1098301511035443

32. Prinja S, Bahuguna P, Faujdar DS, Jyani G, Srinivasan R, Ghoshal S, et al. CostEffectiveness of Human Papillomavirus Vaccination for Adolescent Girls in Punjab State: Implications for India’s Universal Immunization Program. Cancer [Internet]. 2017 Sep;123(17):3253-60. Available from: https://onlinelibrary.wiley.com/doi/full/10.1002/cncr.30734

33. Molina Aguilar IB, Otilia Mendoza L, Garcia O, Diaz I, Figueroa J, Maria Duarte R, et al. Cost-effectiveness analysis of the introduction of the human papillomavirus vaccine in Honduras. Vaccine [Internet]. 2015 May;33(1):A167-73. Available from: https://www.sciencedirect.com/science/article/pii/S0264410X14017228

34. Andres-Gamboa O, Chicaiza L, Garcia-Molina M, Diaz J, Gonzalez M, Murillo R, et al. Cost-effectiveness of conventional cytology and HPV DNA testing for cervical cancer screening in Colombia. Salud Publica Mex [Internet]. 2008;50(4):276-85. Available from: https://www.scielosp.org/scielo.php?pid=S003636342008000400005\&script=sci_arttext\&tlng=pt

35. Campos N, Castle P, Wright T, Kim J. Cervical cancer screening in low-resource settings: A cost-effectiveness framework for valuing tradeoffs between test performance and program coverage. Int J CANCER [Internet]. 2015 Nov;137(9):2208-19. Available from: https://onlinelibrary.wiley.com/doi/full/10.1002/ijc.29594 
36. Levin CE, Sellors J, Shi J-F, Ma L, Qiao Y, Ortendahl J, et al. Cost-effectiveness analysis of cervical cancer prevention based on a rapid human papillomavirus screening test in a high-risk region of China. Int J CANCER [Internet]. 2010 Sep;127(6):1404-11. Available from:

https://onlinelibrary.wiley.com/doi/full/10.1002/ijc.25150

37. Leech AA, Kim DD, Cohen JT, Neumann PJ. Use and Misuse of Cost-Effectiveness Analysis Thresholds in Low- and Middle-Income Countries: Trends in Cost-perDALY Studies. Value Heal [Internet]. 2018 [cited 2019 Oct 9];21(7):759-61. Available from: https://www.sciencedirect.com/science/article/pii/S1098301518300160

38. Simoens S. How to assess the value of medicines? Front Pharmacol [Internet]. 2010 [cited 2019 Oct 9];SEP. Available from: http://journal.frontiersin.org/article/10.3389/fphar.2010.00115/abstract

39. Newall AT, Jit M, Hutubessy R. Are Current Cost-Effectiveness Thresholds for Lowand Middle-Income Countries Useful? Examples from the World of Vaccines. Pharmacoeconomics [Internet]. 2014 Jun 3 [cited 2019 Oct 9];32(6):525-31. Available from: http://link.springer.com/10.1007/s40273-014-0162-x

40. WHO. Comprehensive cervical cancer prevention and control: a healthier future for girls and women [Internet]. Geneva; 2013. Available from: https://www.who.int/cancer/prevention/diagnosis-screening/cervical-cancer/en/

41. Patel C, Brotherton JML, Pillsbury A, Jayasinghe S, Donovan B, Macartney K, et al. The impact of 10 years of human papillomavirus (HPV) vaccination in Australia: What additional disease burden will a nonavalent vaccine prevent? [Internet]. Vol. 23, Eurosurveillance. 2018 [cited 2019 Aug 8]. p. 30-40. Available from: 
https://www.ncbi.nlm.nih.gov/pmc/articles/PMC6194907/

42. Allende G, Surriabre P, Cáceres L, Bellot D, Ovando N, Torrico A, et al. Evaluation of the self-sampling for cervical cancer screening in Bolivia. BMC Public Health [Internet]. 2019 Dec 17 [cited 2019 Aug 9];19(1):80. Available from: https://bmcpublichealth.biomedcentral.com/articles/10.1186/s12889-019-6401-5

43. Yeh PT, Kennedy CE, De Vuyst H, Narasimhan M. Self-sampling for human papillomavirus (HPV) testing: A systematic review and meta-Analysis [Internet]. Vol. 4, BMJ Global Health. 2019 [cited 2019 Aug 9]. Available from: https://gh.bmj.com/content/4/3/e001351?int_source=trendmd\&int_medium=cpc\&int_c ampaign=usage- 042019

44. CDC. HPV Vaccine Recommendations [Internet]. 2019 [cited 2019 Jul 21]. Available from: https://www.cdc.gov/vaccines/vpd/hpv/hcp/recommendations.html

45. Morema EN, Atieli HE, Onyango RO, Omondi JH, Ouma C. Determinants of Cervical screening services uptake among 18-49 year old women seeking services at the Jaramogi Oginga Odinga Teaching and Referral Hospital, Kisumu, Kenya. BMC Health Serv Res [Internet]. 2014 Dec 6 [cited 2019 Aug 9];14(1):335. Available from: http://bmchealthservres.biomedcentral.com/articles/10.1186/1472-6963-14-335

46. Binagwaho A, Wagner C, Gatera M. Achieving high coverage in Rwanda's national human papillomavirus vaccination programme. SciELO Public Heal [Internet]. 2012 [cited 2019 Jul 24]; Available from: https://www.scielosp.org/scielo.php?pid=S004296862012000800014\&script=sci_abstract\&tlng=fr

47. Prue G, Baker P, Graham D, Nutting C, Greenhouse P, Lawler M. It is time for universal HPV vaccination. Lancet (London, England) [Internet]. 2018 Sep 15 [cited 2019 Aug 9];392(10151):913-4. Available from: 
http://www.ncbi.nlm.nih.gov/pubmed/30238882

48. WHO. Human Papillomavirus (HPV) immunization. Geneva; 2018. 


\section{Appendix 1: Search strategy (MEDLINE).}

(((((((((developing countries) AND uterine cervical neoplasms)) AND ((hpv OR human papillomavirus OR papillomaviridae $)))$ AND (((((((screen*) OR test*) OR check*) OR monitor*) OR triag*)) OR ((("visual inspection") OR cytology) OR ((papanicolaou OR pap smear $)))))$ OR ((((((developing countries) AND uterine cervical neoplasms)) AND ((hpv OR human papillomavirus OR papillomaviridae $)))$ AND (((vaccin*) OR immun*) OR innoculat* $))))$ AND $((((($ cost-effective* OR cost effective* $)))$ OR $(($ cost-benefit* OR cost benefit*))) OR ((cost-utility OR cost utility))) AND (hasabstract[text] AND ("2008/01/01"[PDAT] : "2018/12/31"[PDAT]) AND Humans[Mesh]) 


\section{Appendix 2: Search history (MEDLINE)}

\begin{tabular}{|c|c|c|}
\hline S33 & S23 AND S27 & $\begin{array}{l}\text { Limiters - Date of Publication: 20080101- } \\
\text { 20181231; Abstract Available; English } \\
\text { Language } \\
\text { Search modes - Boolean/Phrase } \\
\end{array}$ \\
\hline S32 & S23 AND S27 & $\begin{array}{l}\text { Limiters - Date of Publication: 20080101- } \\
\text { 20171231; Abstract Available; English } \\
\text { Language } \\
\text { Search modes - Boolean/Phrase }\end{array}$ \\
\hline $\mathrm{S} 31$ & S23 AND S27 & $\begin{array}{l}\text { Limiters - Abstract Available; English } \\
\text { Language } \\
\text { Search modes - Boolean/Phrase }\end{array}$ \\
\hline $\mathrm{S} 30$ & S23 AND S27 & $\begin{array}{l}\text { Limiters - Abstract Available; English } \\
\text { Language } \\
\text { Search modes - Boolean/Phrase }\end{array}$ \\
\hline S29 & S23 AND S27 & $\begin{array}{l}\text { Limiters - Abstract Available } \\
\text { Search modes - Boolean/Phrase }\end{array}$ \\
\hline S28 & S23 AND S27 & Search modes - Boolean/Phrase \\
\hline S27 & S24 OR S25 OR S26 & Search modes - Boolean/Phrase \\
\hline S26 & cost-utility or cost utility & Search modes - Boolean/Phrase \\
\hline S25 & cost-benefit* or cost benefit* & Search modes - Boolean/Phrase \\
\hline $\mathrm{S} 24$ & cost-effective* or cost effective* & Search modes - Boolean/Phrase \\
\hline S23 & S17 OR S22 & Search modes - Boolean/Phrase \\
\hline S22 & S5 AND S21 & Search modes - Boolean/Phrase \\
\hline $\mathrm{S} 21$ & S18 OR S19 OR S20 & Search modes - Boolean/Phrase \\
\hline S20 & innoculat* & Search modes - Boolean/Phrase \\
\hline S19 & immun* & Search modes - Boolean/Phrase \\
\hline S18 & vaccin* & Search modes - Boolean/Phrase \\
\hline S17 & S5 AND S16 & Search modes - Boolean/Phrase \\
\hline S16 & S11 OR S15 & Search modes - Boolean/Phrase \\
\hline S15 & S12 OR S13 OR S14 & Search modes - Boolean/Phrase \\
\hline S14 & papanicolaou or papsmear & Search modes - Boolean/Phrase \\
\hline S13 & cytology & Search modes - Boolean/Phrase \\
\hline S12 & "visual inspection" & Search modes - Boolean/Phrase \\
\hline S11 & S6 OR S7 OR S8 OR S9 OR S10 & Search modes - Boolean/Phrase \\
\hline S10 & triag* & Search modes - Boolean/Phrase \\
\hline S9 & monitor* & Search modes - Boolean/Phrase \\
\hline S8 & check* $^{*}$ & Search modes - Boolean/Phrase \\
\hline S7 & test* & Search modes - Boolean/Phrase \\
\hline S6 & screen* & Search modes - Boolean/Phrase \\
\hline S5 & S3 AND S4 & Search modes - Boolean/Phrase \\
\hline S4 & $\begin{array}{l}\text { hpv or human papilomavirus or } \\
\text { Papillomaviridae }\end{array}$ & Search modes - Boolean/Phrase \\
\hline S3 & S1 AND S2 & Search modes - Boolean/Phrase \\
\hline S2 & (MM "Uterine Cervical Neoplasms") & Search modes - Boolean/Phrase \\
\hline S1 & (MM "Developing Countries") & Search modes - Boolean/Phrase \\
\hline
\end{tabular}

